factors (classified high-risk). Knowledge and awareness scores (\% correct) for PVD were low regardless of CV risk factor group $(<50 \%)$. To assess perceived risk, when asked whether they thought they had risk factors for PVD, $53.6 \%$ of moderate-risk women and $58.8 \%$ of high-risk women responded either 'no' or 'not sure'. Discussion: These data demonstrate that women are at an increased risk for both cardiac and non-cardiac vascular diseases, but lack knowledge and awareness of their risk and its implications for both heart and PVDs. We advocate screening programs to detect subclinical vascular and CV disease through the use of surrogate markers such as ABI and cIMT in asymptomatic women, as well as aggressive risk factor management to decrease future adverse event rates. Future longitudinal studies to evaluate the effectiveness of screening tools in the lowering of risk scores are underway.

\section{Abstract PS2-03 \\ Health Communication and Health Literacy for Persons at Risk for CVD - Experiences Using Two Health Care Organizations}

Bridget Gaglio, PhD(c), MPH, Kaiser Permanente - Colorado, Institute for Health Research

Background/Aims: Technological advances in health communications can potentially help or create barriers for individuals to manage chronic disease care. However, we know little about preferences for receiving cardiovascular disease (CVD) prevention information among individuals with multiple risk factors for CVD and varying health literacy levels. The overall design of the study is a mixed-methods design assessing health literacy levels and preferences for receiving CVD prevention information using surveys and in-depth interviews with patients who have two or more risk factors for CVD. However, this presentation will focus on the recruitment process for the study. Methods: Participants were recruited from two health care organizations (an HMO organization and a community health clinic) in Denver, CO in order to obtain a more diverse study sample as well as capture a wider range of health literacy levels and experiences with receiving health information. Using two health care systems required receiving human subjects' approval from two institutional review boards (IRB). The original recruitment methods, which consisted of identifying potentially eligible individuals through each organization's electronic medical records system, sending out an introductory recruitment letter with an opt-out postcard followed up with a recruitment call, were approved by one IRB and not the other. Results: After reassessing the allowable recruitment methods from the community health clinic's IRB, it was decided that the overall recruitment methods for the project would be selected to suit each organization's patient populations. In the HMO setting, the originally proposed methods were used. In the community health care setting, two different methods were used. First, 5 physicians identified patients that were potentially eligible for the study and an 'opt-in' introductory letter was mailed out from each of the physicians. Second, in-clinic recruitment was conducted to reach the final recruitment goal. Experiences with using two different recruitment methods for participants and outcomes associated with using these methods will be reported. Conclusions: The results of these recruitment efforts are important for researchers in that one must consider and plan for potential changes required from IRBs and the implications of such changes on projects when partnering and conducting research with multiple institutions.

Abstract PS2-04

The Potential Impact of Heart Disease Prevention and Treatment Interventions

Thomas E. Kottke, MD, MSPH, HealthPartners Research Foundation; Courtney Jordan, MD, University of Minnesota

Background: Rational policy making could benefit from an estimate of the deaths that might be prevented or postponed (DPP) by developing more effective behavior change programs and fully implementing technologies that are currently available to prevent and treat heart disease. Methods: To estimate the impact of developing more effective behavior change programs and fully implementing currently available technologies to prevent and treat heart disease, we created a hypothetical population ages 30-85 years resembling that of the United States. We divided the population into three prevalence pools (no apparent heart disease, known heart disease without left ventricular systolic dysfunction [LVSD] and known heart disease with
LVSD). We divided clinical events into one of three types: out-of-hospital cardiac arrest, acute or emergent heart disease events, and non-emergent heart disease events. We defined the difference in total DPP between current implementation levels and full implementation levels as the outcome of interest. Results: In this hypothetical population, 87,500 would have no apparent heart disease; 9900 would have heart disease without LVSD; and, 2600 would have heart disease with LVSD. Expected death rates for the three subpopulations would be $0.007,0.035$, and 0.067 , respectively. During 1 year without treatment 1133 individuals would be expected to die: 613 without apparent heart disease, 346 with heart disease but no LVSD, and 174 individuals with heart disease and LVSD. The DPP that would result from implementing lifestyle changes and prescribing medications in the prevalence pools would be 648 and the DPP from implantable defibrillators might be another 60 . The DPP from fully implementing treatments during the three types of acute cardiac events would be 70 . Conclusions: If effective behavior change programs were developed and currently available heart disease prevention and treatment technologies were fully implemented, approximately $62 \%$ of all deaths among adults in the United States might be prevented or postponed. Fully implementing currently available interventions during acute cardiac events would prevent or postpone about $7 \%$ of all deaths.

Abstract PS2-05

Anemia in Heart Failure: Patterns \& Relationships With Health Outcomes

Ella E. Lyons, MS, Institute for Health Research - Kaiser Permanente Colorado; Frederick Masoudi, MD, MSPH, Denver Health and University of Colorado at Denver and Health Sciences Center; Christina Clarke, BS, Institute for Health Research - Kaiser Permanente Colorado; Christina Adams, MD, University of Colorado at Denver and Health Sciences Center; Pamela Peterson, MD, Denver Health; David Magid, MD, MPH, Institute for Health Research - Kaiser Permanente Colorado and University of Colorado at Denver and Health Sciences Center

Background/Aims: The prevalence of anemia in chronic heart failure (HF) is at least one in five patients. Several studies suggest that anemia is independently associated with mortality and hospitalization among patients with HF. Existing studies are often limited by several factors (e.g., inadequate clinical data, non-standardized definitions of anemia), but the most important limitation has been the use of only one laboratory measurement to identify anemia. These studies are thus unable to characterize patterns of anemia over time and their relationship with health outcomes. Within Kaiser Permanente Colorado (KPCO), we identified a 6-year cohort of HF patients. Our aims included 1) describing the incidence and prevalence of anemia, 2) characterizing trajectories of anemia over time, and 3) assessing patterns of anemia over time relative to outcomes of hospitalization and mortality. Methods: To be included in the study cohort, a patient had to be older than 18 years of age with a primary hospital discharge diagnosis of HF (defined as the index event), a KPCO member for at least 6 months prior to the hospitalization, and have at least one hemoglobin (HGB) value during the index event. Patients were classified as anemic at time of index event and at time points during their follow-up period (minimum of 6 months; maximum of 6 years) based on HGB values. Cox proportional hazards analysis was used to assess the independent association between the pattern of anemia and subsequent hospitalization or death. Covariates included baseline HBG value, time dependent comorbidities, serum sodium concentrations, creatinine clearance values, age, and sex. Results: During the 6 year study period, 2478 patients met cohort inclusion criteria. All patients had a baseline HGB value and $90 \%$ had at least one follow-up HGB value. Forty-five percent of patients were determined to be anemic at the index event. Final results will be completed in early January 2008. Conclusions: Preliminary analysis shows that patients with anemia with HF may have poorer outcomes than HF patients who are not anemic. The treatment of HF patients with anemia will be discussed. 